\title{
132 Abordaje de enfermería en heridas de urgencias
}

\section{Nursing management on emergency wound}

\section{R. Fernando García González}

Manuel Gago Fornells

Sol Chumilla López

Victoriana Gaztelu Valdés

Correspondencia:

R. Fernando García González

C/ Castillo de San Marcos, n. ${ }^{\circ} 23$

11500 Puerto de Santa María (Cádiz)

Teléf.: 649956542025

E-mail: fernandogarcia62@gmail.com
Enfermero. UGC Cardiología. Unidad de Hemodinámica. Hospital Universitario de Puerto Real (Cádiz).

Enfermero. Centro de Salud Pinillo Chico. Puerto de Santa María. Distrito Sanitario Cádiz-Bahía La Janda.

Enfermero. UGC Cardiología. Unidad de Hemodinámica. Hospital Universitario de Puerto Real (Cádiz).

Doctora en Medicina y Cirugía. Especialista en Medicina Comunitaria. Centro de Salud Federico Rubio. El Puerto de Santa María (Cádiz).

\section{RESUMEN}

Las heridas ocupan un porcentaje elevado en las demandas de los servicio de urgencias, tanto hospitalario como extrahospitalario. Son lesiones producidas por el efecto de un agente externo que actúa de manera brusca sobre una parte de nuestro organismo, provocando una rotura de la superficie cutánea o mucosa, una discontinuidad en el epitelio que lo reviste. En este artículo queremos abordar la importancia que tiene una valoración inicial de la herida de forma integral, el mecanismo causal que la provocó, así como el tiempo que ha transcurrido desde que se ha producido, factores todos ellos de gran importancia; así como poderla clasificar de forma adecuada para, posteriormente, iniciar los cuidados correctos en función de las características de cada paciente y de la propia herida. Muchos son los elementos que cobran importancia en el proceso de cicatrización de la propia herida, además de una serie de factores que van a hacer que este proceso de reparación se vea mermado por múltiples razones.

\section{PALABRAS CLAVE}

Herida aguda, cicatrización de heridas, clasificación de heridas.

\section{SUMMARY}

Wounds occupy a high percentage in the demands of the emergency service, both inpatient and outpatient, are lesions caused by the effect of an external agent acting abruptly over a part of our body, causing a skin surface or mucosa breakdown, a discontinuity in the epithelium it takes. In this article we focus on the importance of an initial integral assessment of the wound: causal mechanism that caused it and time that has elapsed since the occurrence, as both are of great importance. Also, to properly classify the wound, for later initiate correct care according to the characteristics of each patient and the wound itself. Many are the elements become important in the healing process of the wound itself and a series of factors that are going to make this repair process will be reduced through multiple reasons.

\section{KEYWORDS}

Acute wound, wound healing, wound classification.

\section{INTRODUCCIÓN}

Una herida es el efecto producido por un agente externo que actúa de manera brusca sobre una parte de nuestro organismo, superando la resistencia de los tejidos sobre los que incide, causando una rotura de la superficie cutánea o mucosa, ya sea producida accidentalmente por traumatismos $\mathrm{u}$ otras agresiones a la piel, o por un acto quirúrgico. En definitiva, es una lesión caracterizada por una discontinuidad en el epitelio que lo reviste.

Actualmente, el profesional de enfermería se enfrenta a diario a diferentes situaciones relacionadas con las heridas agudas; sin duda, la infección es una de ellas e indudablemente de las de mayor impacto. La lucha por la infección deberíamos plantearla mediante un abordaje interdisciplinar, haciendo especial hincapié en el exhaustivo lavado de manos, adecuado instrumental a utilizar así como la correcta realización de los distintos procedimientos con las medidas asépticas adecuadas y abordaje de la herida, sin olvidar la cura adecuada y el material idóneo.

Todas estas son pues las piedras angulares para una buena prevención, ya que el correcto seguimiento de todos estos procesos en el cuidado de la herida va a reducir las tasas de infección y sus complicaciones, además de disminuir el tiempo de hospitalización de los pacientes con los consiguientes 
a

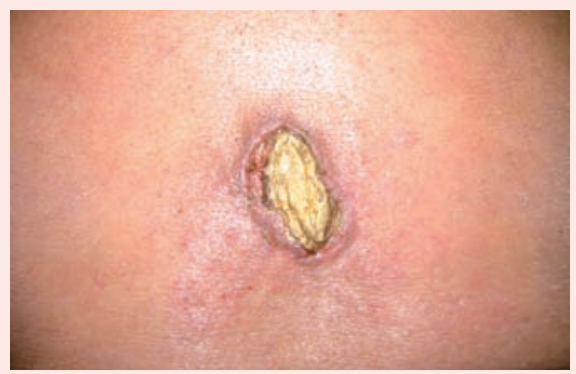

b

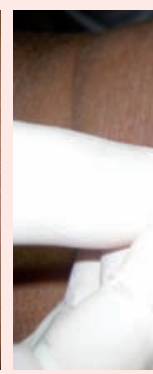

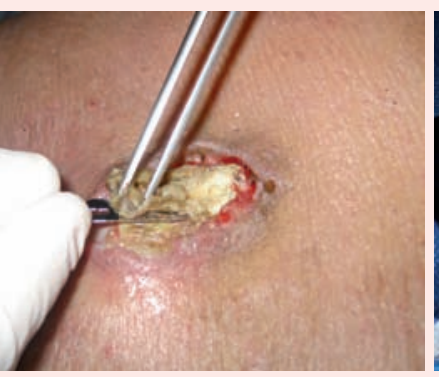

C

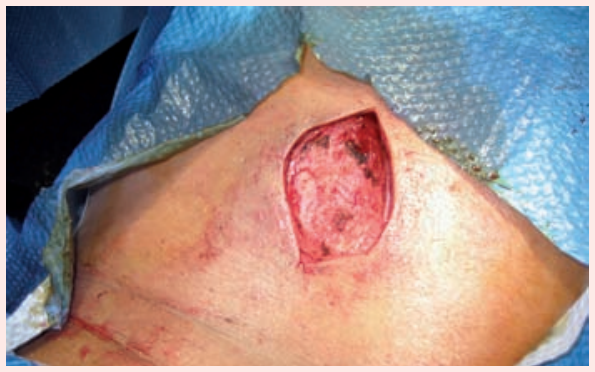

Fig. 1. a) Cuerpo extraño. Restos de gasa en lecho de herida. b) Desbridamiento cortante. c) Resultado final tras desbridamiento quirúrgico (quirófano).

ahorros de recursos, no solo materiales sino también de humanos $(1,2)$.

Las heridas agudas, a diferencia de las heridas crónicas, normalmente tienen un proceso de reparación dinámico, ordenado y escalonado que concluye con la restauración continua de la integridad de la piel, pero hay elementos que pueden interferir en el proceso de cicatrización $(3,4,5,6,7)$.

El profesional de enfermería debe conocer que el proceso de cicatrización no es igual en todos los pacientes y que varía de acuerdo con la vulnerabilidad a reacciones indeseables, las cuales dependen de un grupo de factores locales y generales; además, debe aplicar los principios importantes en el cuidado de las heridas, que lo guiarán en la atención al paciente al poder seleccionar la alternativa terapéutica conveniente en el tratamiento local y cuidados posteriores $(8,9)$.

\section{FACTORES QUE AFECTAN A LA CICATRIZACIÓN DE LA HERIDA}

La curación de heridas, como un proceso biológico normal en nuestro cuerpo, se logra a través de cuatro fases con precisión y altamente programadas: hemostasia, inflamación, proliferación y remodelación. Para que una herida se cure correctamente, las cuatro fases deben producirse en la secuencia adecuada y dentro de un tiempo determinado; esta serie de eventos biológicos establecidos intentan devolver la integridad anatómica, funcional y estética de los tejidos lesionados, dejando finalmente una cicatriz. Pero hay factores que pueden interferir con una o más fases de este proceso, causando así la curación de heridas de manera inadecuada o dañada $(10,11)$.

\section{Factores locales}

Son los que influyen directamente en las características de la herida, mientras que los factores sistémicos se relacionan con el estado general de salud o estado de la enfermedad del individuo y que afectan a su capacidad de curación.

Muchos de estos factores están relacionados y los factores sistémicos que actúan a través de los efectos locales que afectan a la cicatrización de heridas.

\section{Oxigenación de la zona}

Cualquier elemento que impida o disminuya la circulación de la sangre en la zona de una herida retarda su cicatrización y la hace más vulnerable a infecciones, como ocurre en ocasiones con determinados vendajes apretados, arterias dañadas, etc. Aunque se ha comprobado que un pequeño grado de edema estimula la fibroplasia, formando parte del tejido de granulación, cuando es considerable retrasa la cicatrización e inhibe el transporte al área de las sustancias necesarias para este proceso. En resumen, el nivel de oxígeno adecuado es crucial para la curación de las heridas. La hipoxia y la isquemia son las causas más frecuentes del retraso de la cicatrización, provocando la depleción de los bordes de la herida, dificulta la síntesis de colágeno, impide la migración de fibroblastos, y reduce los mecanismos de defensa contra la invasión bacteriana, aumentando la susceptibilidad de la herida a la infección. En general, la hipoxia es el mecanismo común por el que otros factores dificultan la cicatrización, siendo el único proceso de cicatrización favorecido por la hipoxia la angiogénesis. Una opción terapéutica que a veces puede superar la influencia de la hipoxia tisular es la terapia de oxígeno $(12,13,14)$.

\section{Infección}

Una vez lesionada la piel, los microorganismos que están normalmente secuestrados en la superficie de la piel tienen acceso directo a los tejidos subyacentes. El estado del individuo y su respuesta ante la invasión de microorganismos determinan si la herida se clasifica como: teniendo contaminación, colonización, infección local / colonización crítica o propagación de la infección invasiva $(15,16)$.

\section{Cuerpos extraños}

$\mathrm{Al}$ igual que la infección, los procesos infecciosos pueden causan destrucción tisular y en consecuencia, retrasar o anular el proceso de cicatrización. En ocasiones, una mala exploración de la herida nos deja pasar por alto elementos o detritus que quedan alojados en el interior tales como cristal, hueso, madera, espinos, etc., y procedemos a la sutura de la misma con la consiguiente dehiscencia quirúrgica posterior (Fig. 1).

\section{Movilidad de los tejidos}

La inmovilidad de los bordes de la herida es muy importante para adelantar la reparación y curación de las heridas, sobre todo en tejidos profundos como músculos y tendones. Por eso, se recomienda el reposo durante los primeros días, y si el trauma es en los miembros inferiores o en articulaciones los inmovilizaremos.

\section{Extensión de la destrucción hística}

Es otro factor determinante de la reparación, más prolongado cuando el daño tisular es más extenso.

\section{Tejido donde ha ocurrido la lesión}

Algunos tejidos tienen más inmunidad que otros. La vascularización influye de manera notable en este proceso de aceleramiento en la cicatrización; el tejido muy vascularizado está menos expuesto a infecciones que el que está irrigado por una circulación más limitada. Zonas como el perineo es posible que retarden más su cicatrización por las condiciones de humedad de la zona que otras que no estén expuestas a este elemento.

\section{Edema}

Reduce el riego sanguíneo a raíz del aumento de la presión intersticial sobre los vasos sanguíneos; paraliza la cicatrización al inhibir los elementos necesarios en este proceso de formación ya que disminuye el $\mathrm{O}_{2}$ en los tejidos, aumentando el $\mathrm{CO}_{2}$, haciendo más ácido el medio y perturba el proceso regenerativo.

\section{Radioterapia}

Se retrasa, la cicatrización, en pacientes que se han sometido a radioterapia, no solo por la inmunosupresión, sino también por el daño tisular extenso en el órgano o región 
Tabla 1. Clasificación en función de la complejidad, etiología, forma y contaminación de la herida (clasificación del autor)

\begin{tabular}{|l|l|l|l|}
\hline \multicolumn{1}{|c|}{ Complejidad } & \multicolumn{1}{|c|}{ Forma } & \multicolumn{1}{c|}{ Etiología } \\
\hline Longitudinales & Lineales & Punzantes \\
Transversas & Curvas & Incisas & Limpias \\
Oblicuas & En colgajo & Contusas \\
Espiroideas & Estrelladas & Por arma de fuego \\
Simples: epidérmicas, arañazos, & En Scalp & Por arma blanca \\
desolladuras & Con pérdida de sustancia & Por aplastamiento \\
Complejas: penetrantes, & Arrancamiento & Por asta de toro \\
perforantes, empalamientos & Amputación & Por agente químico \\
& & Por agente térmico \\
& & Por mordedura \\
\end{tabular}

del cuerpo tratados. En pacientes radiados suele complicarse la cicatrización de heridas, incluyendo retraso, dehiscencia y fístulas. No olvidemos que los tejidos radiados presentan alteraciones en el metabolismo celular y deben manipularse con mayor delicadeza.

\section{Tejidos desvitalizados}

Es otro de los elementos que jugará un papel importantísimo en el proceso de reparación de la propia herida, por lo que se hace imprescindible su eliminación para garantizar la reparación hística.

\section{Hematomas}

Se trata de otro elemento determinante, llegando a separar los tejidos profundos, limitando el aporte sanguíneo. La propia tensión expande la piel para acomodar el volumen del hematoma. Las necrosis cutáneas, generalmente, ocurren al no detectar hematomas. Los grandes deben ser drenados. Y los pequeños desaparecerán con evacuaciones seriadas. La hemostasia meticulosa, la disección juiciosa del colgajo y la atención al dolor posoperatorio son las mejores defensas para evitar estas complicaciones (Fig. 2).

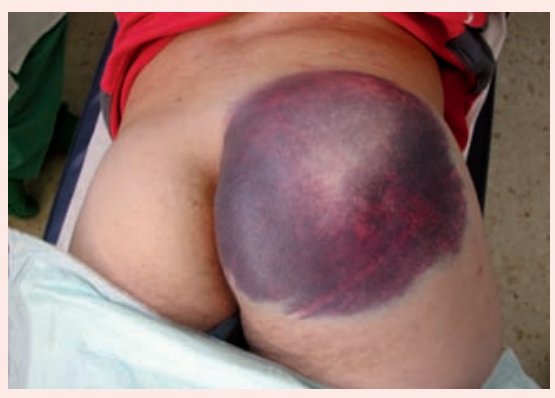

Fig. 2. Hematoma postraumático.

\section{Curaciones repetidas}

La repetición de las curas a pequeños intervalos de tiempo puede perjudicar el proceso de cicatrización debido a la remoción de los elementos celulares por la propia gasa o instrumental.
Tensión en los bordes de la berida

En heridas suturadas, los puntos deben ejercer la misma tensión entre los bordes de la herida que entre la superficie y el fondo de esta. A esta norma se le denomina regla de las x (equilibrada, equidistante, equipenetrante) como refieren Domínguez Romero y cols. (17).

Factores generales

\section{La edad}

En los ancianos, la circulación suele estar deteriorada por arteriosclerosis y una función cardiaca limitada. Sus reservas de energía son bajas, y con frecuencia su hidratación y estado nutricional deficientes. La epidermis es más fina y su renovación más lenta, disminuyendo la resistencia al traumatismo (18).

\section{El Estado nutricional}

La nutrición de un individuo tiene un gran efecto en el proceso de cicatrización. Las proteínas son fundamentales en la formación de nuevo tejido. Las deficiencias proteínicas retrasan la vascularización, la formación de linfáticos, la proliferación de fibroblastos, la síntesis de colágeno y la remodelación de la herida. Los carbohidratos y las grasas se requieren para la energía celular. La vitamina C participa en la maduración de las fibras de colágeno durante las últimas etapas de la cicatrización. La carencia de vitamina K puede causar hemorragias y hematomas que hacen más difícil la cicatrización. Las vitaminas del complejo B son necesarias para el metabolismo de proteínas, grasas y carbohidratos, aunque no se conoce el mecanismo. En pacientes obesos, es mayor la frecuencia de complicaciones de heridas, como hernias incisionales, infecciones y dehiscencias (19, $20,21,22$ ).

\section{Determinadas enfermedades}

Algunas como las autoinmunes, personas con anemia y trastornos sanguíneos que causan insuficiencia vascular donde se disminuye la resistencia del paciente y aumenta la posibilidad de infección. Los AINE y los corticoides reducen la fase de inflamación necesaria en el proceso de cicatrización. Además, los corticoides actúan como inmunodepresores y disminuyen la actividad de los leucocitos, por lo que aumenta el riesgo de infección, tratamientos con dicumarínicos y antiagregantes plaquetarios $(23,24)$.

\section{CLASIFICACIÓN}

Las heridas agudas tienen una amplia gama de clasificaciones. Para muchos autores (25, 26) las podemos clasificar en función de varios aspectos, atendiendo a diversas características de las mismas, como la forma de la herida, la profundidad y afectación de estructuras adyacentes, el tamaño, el mecanismo de producción, la complejidad, el grado de contaminación, etc. Es fundamental describir de forma detallada el aspecto, forma, agente causal y afectación de las distintas capas de la piel, ya que de ello depende nuestra actitud terapéutica y cuidados posteriores de la propia herida $(27,28,29)$ (Tabla 1).

1. Según la dirección o trayecto de la herida o zona del cuerpo donde se ha producido la herida, pueden ser:

- Longitudinales. Cuando el trayecto de la herida sigue un curso más o menos paralelo al eje principal siguen un trayecto recto, eje longitudinal mayor que el transversal. Se producen en zonas donde la tensión estática de la piel es muy fuerte, la piel de la frente o en zonas con grandes masas musculares debajo (pierna, brazo); la parte central de la herida tiende a separarse y se retraen los bordes (Figs. 3 y 4 ).

- Transversales. Donde los bordes cutáneos de la herida no siguen las líneas de tensión de la piel.

- Oblicuas. Similares a las transversales. La diferencia está en el ángulo que for- 


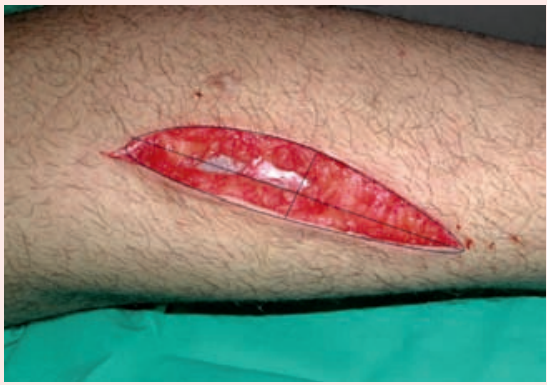

Fig. 3. Herida longitudinal.

ma la línea imaginaria que sigue el corte de los planos de la piel causada por la herida con las líneas de adherencia de esta.

- Espiroideas. Producidas por contusiones, la fuerza aplicada por el agente causante provoca compresión e incluso tensión en el foco traumático. En este tipo de heridas se encuentran diferentes direcciones del eje mayor en la misma lesión, lo que unido al mecanismo causante complica la reconstrucción y el resultado estético.

2. Según la profundidad de la propia herida, y dependiendo de la afectación que esta ha tenido en los distintos planos de la piel (30) las podemos clasificar en:

- Superficiales o simples. Dentro de estas entrarían todas las que solo afectan a la epidermis, sin afectar a elementos nobles (vasos, nervios, tendones) como las epidérmicas, arañazos o desolladuras cuando conlleva cierta pérdida de tejido epitelial.

- Profundas. Cuando se extienden más allá del tejido celular subcutáneo afectando a varios tejidos, piel, músculo o huesos donde la reconstrucción y regeneración será más dificultosa (Figs. 5 y 6).

- Penetrantes. Cuando alcanzan una cavidad natural del organismo, como puede ser la cavidad peritoneal o el espacio pleural, sin lesionar vísceras u órganos (Fig. 7).

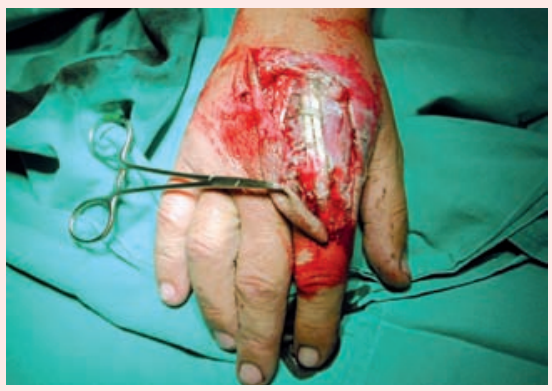

Fig. 6. Tendones expuestos.

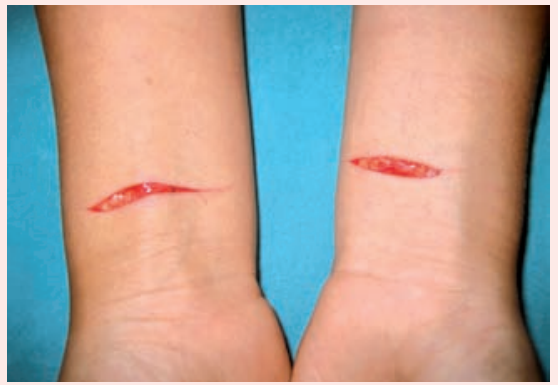

Fig. 4. Heridas longitudinales.

- Perforantes. Afectan a las cavidades, penetrando y llegando a lesionar.

- Por empalamiento. La causada por un objeto inciso- punzante, de forma que el objeto queda atrapado en el cuerpo del sujeto o en alguno de los orificios naturales del organismo, provocando diferentes tipos de lesiones y afectaciones en distintos órganos que se ven afectado $(31,32,33,34)$.

3. Según la forma de la propia herida, hablaríamos de lineales, curvas, arqueadas, estrelladas, puntiformes, crateriformes y para aquellas que no se ajustan a ninguna de estas formas, de heridas irregulares. Aquellas en las que existe un amplio despegamiento de la piel manteniendo su vascularización a expensas de un puente o pedículo, las denominaremos heridas con colgajo, cuando afectan al cuero cabelludo se denominan Scalp (Fig. 8).

Es por eso que se escucha también la expresión scalp pretibial para hacer referencia a heridas en la pierna que afectan a todo el espesor de la piel y exponen estructuras nobles. Lo correcto, por ejemplo, sería hablar de avulsión pretibial o herida en colgajo. Por último, hablábamos de heridas con pérdida de sustancia cuando existe una importante pérdida de tejidos en el lecho de la herida (Fig. 9).

4. Según el agente etiológico o mecanismo por el que se producen, siendo esta clasificación la más relevante desde un punto de vista pronóstico y terapéutico.

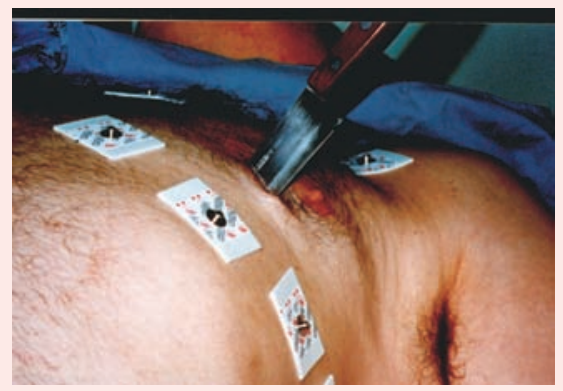

Fig. 7. Arma blanca.

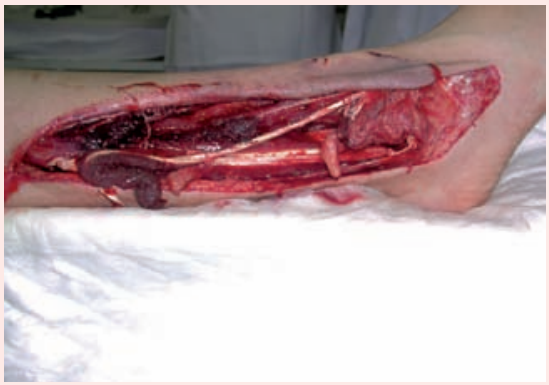

Fig. 5. Accidente de moto.

Cuando la herida se produce por la actuación de un cuerpo puntiagudo de forma más o menos cónica, hablamos de heridas punzantes, predominando la profundidad sobre la extensión de la herida. Estos pequeños agujeros en la piel pueden ocultar una lesión grave a la vez que sus márgenes presentan un grado variable de daño que es debido a la compresión que el agente traumático produce durante su penetración (Figs. 10 y 11).

Es importante observar en dichos bordes derrames sanguíneos, así como tejidos contusionados, cuya viabilidad puede estar más o menos comprometida al atravesar diferentes planos tisulares dotados cada uno de ellos de unas características elásticas y/o contráctiles diferentes. El trayecto producido por el agente pierde sus características topográficas tras la retirada de este, convirtiéndose en un trayecto irregular y anfractuoso en el que con facilidad pueden almacenarse líquidos y restos de tejidos que favorezcan la proliferación bacteriana.

Las producidas por agentes cortantes y/o bordes muy afilados son las llamadas incisas. En ellas predomina la longitud sobre las otras dimensiones; con bordes limpios y suelen sangrar mucho (Figs. 12 y 14).

Cuando el agente es romo, además de la solución de continuidad propia de la herida, nos encontraremos con un grado más o menos importante de contusión en los tejidos con bordes contundidos y aplastados denominándose contusa; en estos casos, la reparación de la herida va a ser más

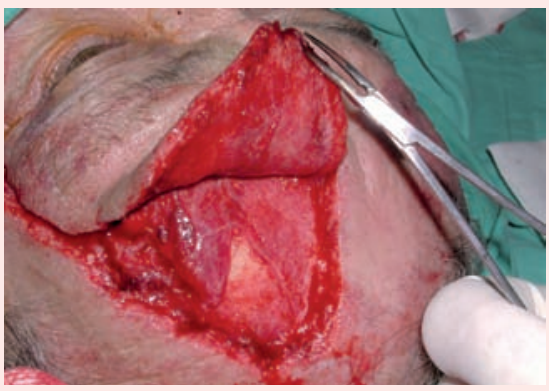

Fig. 8. Scalp. 


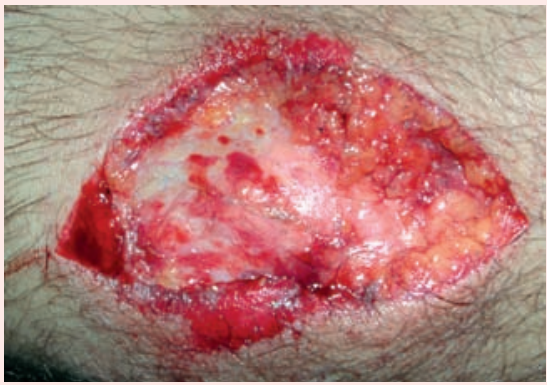

Fig. 9. Pérdida de sustancia en la rodilla.

dificultosa. Las heridas por arrancamiento son producidas por tracción violenta sobre tejidos; suelen ser irregulares y con bordes despegados. Las heridas abrasivas afectan a la epidermis y se producen por fricción.

Por último, las heridas por mordedura, de especial importancia por el riesgo de infección que conllevan; las heridas por arma de fuego, donde el orificio de entrada es menor que el de salida; este último es irregular y de bordes evertidos; las heridas por aplastamiento que en principio pueden aparecer como lesiones superficiales sin interés y dar lugar a un importante síndrome compartimental; finalmente, las de asta de toro (31), de gran importancia y que requerirían un apartado específico debido a su complejidad y las características que suelen presen$\operatorname{tar}(35)$.

5. Según la carga bacteriana (Tabla 2) de la propia herida o grado de contaminación $(36,37,38,39,40)$.

\section{ABORDAJE INICIAL DE LAS HERIDAS. PUNTOS CLAVES}

1. La exploración y valoración la realizaremos con buena iluminación y con las mayores medidas de asepsia posibles (41). Valoraremos la presencia de hemorragia, localización, forma, extensión y longitud de la herida, aspectos de los bordes, limpios o contusos, así como la profundidad, posibles afectaciones de estructuras como nervios tendones o ligamentos y/o determinar el compromiso anatómico subyacente previa anestesia de la zona, grado de suciedad, cuerpos extraños, grado de contaminación, por el tipo del agente traumático y el tiempo transcurrido desde la lesión hasta el tratamiento. El dolor juega un papel importante y a tener en cuenta previo a la exploración, ya que vamos a acceder a zonas hiperestésicas y no olvidemos que el umbral del dolor en todas las personas no es igual, por ello, es de vital importancia la infiltración anestésica previa y analgesia (42).

Como norma general, el límite de tiempo para realizar una sutura primaria es
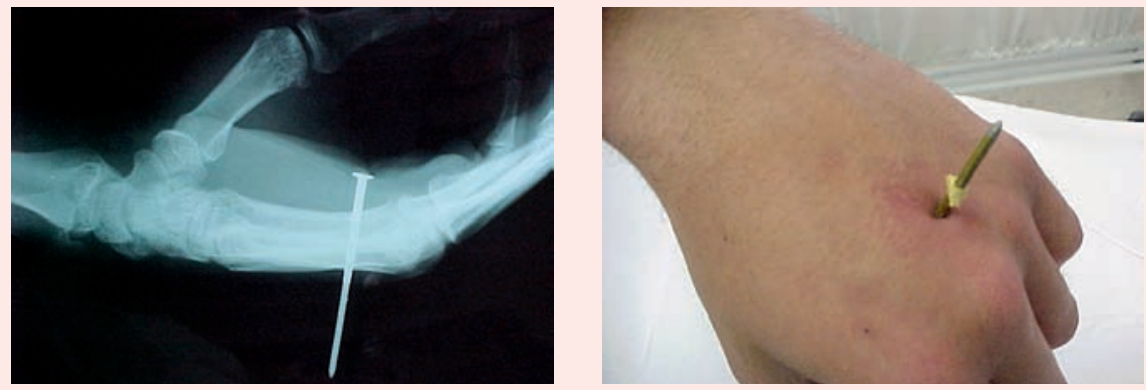

Fig. 10. Clavo en la mano.

Fig. 11. Clavo en la mano.

de seis horas. Un tiempo superior a seis horas obliga a cicatrización por segunda intención, salvo en zonas muy vascularizadas como la cara por el daño estético y el cuero cabelludo en que se puede retrasar hasta 12 o 24 horas. La explicación de todo el procedimiento será de vital importancia para el paciente, además del aporte de seguridad que le vamos a dar.

2. El condicionamiento y el mecanismo de producción juega un papel fundamental para el abordaje posterior de la herida. El lugar y en qué condiciones se ha producido, si ha sido un lugar limpio o sucio, con características de alto riesgo tetanígeno o gran cantidad de material que puedan contener esporas y/o que presenten grandes zonas de tejido desvitalizado lo que abre la posibilidad de vacunación del individuo. Todo ello forma parte de la anamnesis que se le realizará al afectado así como antecedentes de alergias.
3. La inmovilización de la zona afectada la haremos, sobre todo, cuando se trate de un miembro por la posibilidad de que se haya presentado de forma paralela una fractura, además de reducir de forma considerable el dolor. En el caso de transferir al paciente cubriremos con gasas que se aplicaran sobre la herida directamente, manteniendo cierta humedad con suero salino al $0,9 \%$ para, de esta forma, evitar que se pegue esta a la herida; evitaremos gasas vaselinadas ya que maceran los tejidos.

Finalmente, fijaremos con un vendaje, también de gasa y almohadillado que ofrezca una compresión suave complementada por una férula de yeso para inmovilizar la muñeca o el tobillo. En heridas de las manos y pie, las gasas se aplicaran separando los dedos.

4. La limpieza de la herida la realizaremos con solución salina al 0,9\% mediante

\section{Tabla 2. Según la carga bacteriana \\ Clase I. Herida limpia \\ (La frecuencia de infección puede oscilar entre el 5\%-10\%) \\ Herida limpia tiene entre un $1 \%$ a $5 \%$ de desarrollar infección del tejido profundo. \\ La herida es cerrada por primera intención. Las heridas con sistemas de drenaje cerrados entran en esta categoría. Las heridas incisionales operatorias que se realizan luego de trauma contuso se incluyen en esta categoría}

Clase II. Herida limpia-contaminada $(<10 \%)$

Limpia / contaminada tiene entre 4\%-10\% de riesgo de infección

Clase III. Herida contaminada (20\%)

Contaminada tiene un riesgo $>10 \%$ de probabilidad de infección, incluso con profilaxis antibíotica

Clase IV. Herida sucia/infectada $(<40 \%)$

Aumenta el riesgo de infección a un $27 \%$

Tomado de Devaney, Lynn, y cols. Improving Surgical Wound Classification-Why it Matters, AORN (2004). 80: $208-223$ 


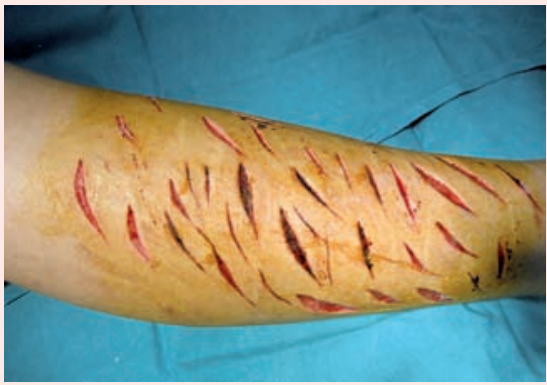

Fig. 12. Heridas incisas.

chorro a presión, para lo cual se puede usar una jeringa de $20 \mathrm{ml}$ con aguja; preferiblemente suero tibio a $37^{\circ} \mathrm{C}$ para evitar el enfriamiento del tejido y mantener la temperatura corporal, además de que es una solución isotónica y no interfiere con el proceso de cicatrización normal, no daña el tejido, no causa sensibilización o alergias y no altera la flora bacteriana normal de la piel (lo que permitiría el crecimiento de microorganismos más virulentos) (43, 44, 45, 46). El agua corriente también se recomienda (47), ya que es eficiente, no habiendo diferencias significativas en comparación con la solución salina $0,9 \%$ (48, 49, 50, 51, 52).

Los antisépticos más utilizados son los derivados yodados y la clorhexidina, sin olvidar el agua oxigenada en la limpieza de las heridas anfractuosas por la presencia de gérmenes anaerobios $(53,54,55,56,57)$.

5. La profilaxis antitetánica no debe obviarse bajo ningún concepto. Las heridas, especialmente las contaminadas con tierra, profundas, punzantes o anfractuosas, son el caldo de cultivo ideal para el crecimiento del bacilo. El tétanos es una enfermedad aguda causada por una exotoxina (tetanospasmina) del Clostridium tetani (bacilo Gram positivo, anaerobio), micro-

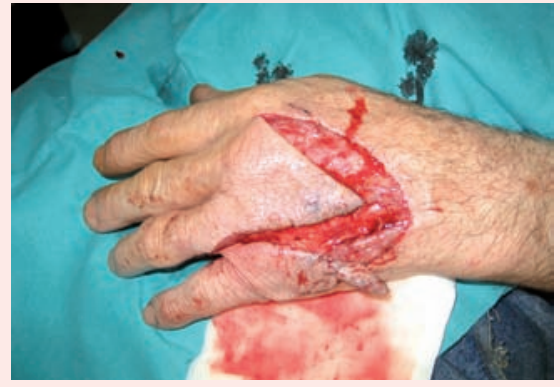

Fig. 13. Herida contusa.

organismo ubicuo en la naturaleza, por lo que no es una enfermedad erradicable; el periodo de incubación oscila generalmente entre tres días y tres semanas desde la inoculación del microorganismo, aunque en ocasiones pueden transcurrir hasta meses.

Distinguimos las heridas de alto riesgo de las de bajo riesgo (Tabla 3):

- Heridas de alto riesgo. Son aquellas con más de seis horas de evolución, anfractuosas, con una profundidad de más de un centímetro, con tejido no viable y contaminante (cuerpos extraños, saliva, tierra, abonos), las producidas por armas de fuego, quemaduras, congelación, asta de toro...

- Heridas de bajo riesgo. Son aquellas con menos de seis horas de evolución, producidas por corte limpio, incisas, con menos de $1 \mathrm{~cm}$ de profundidad, de fondo sangrante y sin presencia de cuerpos extraños.

6. Cuidados posteriores, las curas se realizarán y espaciarán dependiendo de la evolución de la herida, se le darán al paciente las recomendaciones acerca del cuidado de la propia herida, vigilancia del tipo de apósito, presencia de fiebre o escalofríos, enrojecimiento de la zona así como diferentes pautas de actuación.

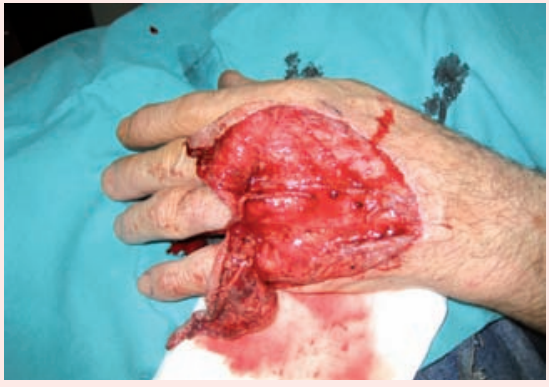

\section{BIBLIOGRAFÍA}

1. Cohen IK, Diegelman RF, Dome RY, y cols. Wound care and wound healing. In: Schwartz SI, Shires GT, Spencer FC, y cols., editors. Principles of surgery. seventh edition. New York: McGrawHill, 1999, pp. 263-95.

2. European Wound Management Association (EWMA). Documento de Posicionamiento: Heridas de difícil cicatrización: un enfoque integral. Londres: MEP Ltd, 2008.

3. Lee ChK, Hansen SL. Tratamiento de las heridas agudas. En Surg Clin N Am 2009; 89: 65976.

4. Prevención y control de la infección de herida quirúrgica. Consultado 21 de diciembre de 2012. Disponible: http://www.mpsp.org/mpsp/Documentos/inf_nosoc/heridaq.htm

5. Cutting KF, White RJ. Revisión de criterios para la identificación de infecciones en heridas. Gerokomos 2006; 17: 39-47.

6. White RJ, Cooper R, Kingsley A. Wound colonization and infection: the role of topical antimicrobials. British Journal of Nursing, 2001, vol. 10 n. ${ }^{\circ} 9$.

7. Wysocki AB (1992) Skin. In: Bryant RA ed. Acute and Chronic Wounds. Nursing Management. London. Mosby Year Book.

8. Rovina E, Candel E. Urgencias en Enfermería, Urgencias traumáticas y desequilibrios con el medio ambiente, Heridas y Contusiones. 2000; 64: 254264.

9. American College of Surgeons: Care of the Surgical Patient, Vol. I, Scientific American, New York, 1999.

10. Gosain A, DiPietro LA. Aging and wound healing. World J Surg 2004; 28: 321-6.

11. European Wound Management Association (EWMA). Documento de Posicionamiento: Heridas de difícil cicatrización: un enfoque integral. Londres: MEP Ltd, 2008.

12. RodrÍguez PG, Felix FN, Woodley DT, Shim EK. The role of oxygen in wound healing: a re-

\begin{tabular}{|c|c|c|c|c|}
\hline \multirow{2}{*}{$\begin{array}{l}\text { Antecedentes de } \\
\text { vacunación }\end{array}$} & \multicolumn{2}{|l|}{ Herida limpia } & \multicolumn{2}{|l|}{ Herida tetanígena $^{a}$} \\
\hline & Vacuna (Td) & $\mathrm{IGT}^{\mathrm{b}}$ & Vacuna (Td) & $\mathrm{IGT}^{\mathrm{b}}$ \\
\hline$<3$ dosis o desconocida & Sí (completar vacunación) & No & Sí (completar vacunación) & Sí \\
\hline 3 o 4 dosis & $\begin{array}{l}\text { No (si hace más de } 10 \text { años desde la última } \\
\text { dosis, administrar una dosis) }\end{array}$ & No & $\begin{array}{l}\text { No (si hace más de cinco años desde la última } \\
\text { dosis, administrar una dosis) }\end{array}$ & $\mathrm{No}^{1}$ \\
\hline 5 o más dosis & No & No & $\begin{array}{l}\text { No (si hace más de } 10 \text { años desde la última dosis, } \\
\text { valorar la administración de una única dosis adi- } \\
\text { cional en función del tipo de herida) }\end{array}$ & $\mathrm{No}^{1}$ \\
\hline
\end{tabular}


view of the literature. Dermatol Surg 2008; 34: 159-69.

13. Bishop A. (2008). Role of oxygen in wound healing. J Wound Care 17: 399-402.

14. Bilevich E. Cicatrización de heridas. Un enfoque práctico, Forum de Flebología y Linfología 2007; 9: 30-6.

15. Edwards R, Harding KG.. Bacteria and wound healing. Curr Opin Infect Dis 2004; 17: 91-6.

16. European Wound Management Association (EWMA). Position Document: Management of wound infection. London: MEP Ltd, 2006.

17. Domínguez Romero M. Galiana Martínez J A. Pérez Vega F J. (2002) Manuel de cirugía menor. Editorial Aran.

18. Keylock KT, Vieira VJ, Wallig MA, DiPietro LA, Schrementi M, Woods JA. Exercise accelerates cutaneous wound healing and decreases wound inflammation in aged mice. Am J Physiol Regul Integr Comp Physiol. 2008; 294: R179-84. Epub 2007, Nov 14

19. Wilson JA, Clark JJ. (2004). Obesity: impediment to postsurgical wound healing Adv Skin Wound Care 17: 426-35.

20. Heyman H, Van De Looverbosch DE, Meijer EP, Schols JM. Benefits of an oral nutritional supplement on pressure ulcer healing in long-term care residents. J Wound Care. 2008; 17: 476-8.

21. Wozniak SE, Gee LL, Wachtel MS, Frezza EE. Adipose tissue: the new endocrine organ? A review article. Dig Dis Sci 2009; 54: 1847-56.

22. Gray D, Cooper P. Nutrition and wound healing: what is the link? Wound Care 2001; 10: 86-9.

23. Brem H, Tomic-Canic M. Cellular and molecular basis of wound healing in diabetes. J Clin Invest 2007; 117: 1219-22.

24. Huijberts MS, Schaper NC, Schalkwijk CG. Advanced glycation end products and diabetic foo disease Diabetes Metab Res Rev 2008; 24 Suppl 1: $S 19-24$

25. Kumar S, Leaper DJ. Classification and management of acute wounds British Journal of Nursing, 2001; 10: 563-578.

26. Lee ChK, Hansen SL. Tratamiento de las heridas agudas. Publicado en Surg Clin N Am 2009; 89: 659-76, vol. 89, n. 03

27. M, Gómez Bravo F. "Principios Básicos en el Tratamiento de las Heridas”. Manual Práctico de Urgencias Quirúrgicas del Hospital Universitario 12 de Octubre 1998.

28. Arribas Blanco JM, Artuñedo PP, Caballero Martínez F. "Tratamiento de las heridas que precisan sutura”. Manual de cirugía menor y otros procedimientos en la consulta del médico de familia. Madrid, 1993
29. Ferreira MC, Tuma Jr. P, Carvalho VF, Kamamoto F. Complex wounds. Clinics. 2006; 61: 571-8.

30. Otero Cacabelos M. Heridas: diagnóstico y tratamiento de urgencia. Consultado 21 de diciembre de 2012.http://www.semg.es/revista/abril2000/379384.pdf.

31. Guerra S. Actitud prehospitalaria ante el paciente empalado. Puesta al día emergencias y catástrofes 2000; 3: 168-73.

32. Lasagna R, Lasagna N, Farin̄a C, Fariña L. Empalamiento transabdominal. Rev Chil Cir 2002; 4: 408-10.

33. Yavrouian R, Mahmoud A, Matolo N. Abdominal impalement resulting in an isolated duodenal injury. Surg Rounds 2008; 2: 59-61.

34. Morales JL, Arriagada R, Gómez J, Santana R, Martel K, Morales C. Empalamiento tóraco-abdominal: Reporte de un caso. Rev. Chilena de Cirugía. Vol. 61 - No 4, Agosto 2009; pp. 3669.

35. García González RF, Martínez García P, Gago Fornells M. Herida por asta de toro. Equipo médicoquirúrgico interdisciplinar y actuaciones en enfermería Puesta al día en urgencias, emergencias y catástrofes, ISSN 1576-0316, vol. 4, n.o 4, 2003, pp. 186-190.

36. Alorda Terrasa C. Cura de una herida limpia. Cura de una herida contaminada o infectada. Metas de enfermería. N.o 27. pp. 14-8. Julio/agosto. 2000.

37. Gil P, Esteban E, Legido P, Gago P, Pastor E. Infection rate in clean surgery: follow-up until 30 days after operation. Cir Esp. 2005 Apr; 77: 226-

38. Knight R, Charbonneau P, Ratzer E, Zeren F, Haun W, Clark J. Prophylactic antibiotics are not indicated in clean general surgery cases. Am J Surg. 2001 Dec; 182: 682-6.

39. Lilani SP, Jangale N, Chowdhary A, Daver GB. Surgical site infection in clean and clean-contaminated cases. Indian J Med Microbiol. 2005 Oct; 23: 249-52

40. World Health Organization. Regional Office for South-East Asia. Guidelines on Prevention and Control of Hospital Associated Infections. World Health Organization. Regional Office for SouthEast Asia. New Delhi. January 2002.

41. Hollander JE, Singer AJ. Evaluación de las heridas. En: Tintinalli JE, GD Kelen, JS Stapczynski, DO Ma, DM Cline, eds Emergency Medicine:. Guía de Estudio Integral. $6^{\text {a }}$ ed. Columbus, $\mathrm{OH}$ : McGraw-Hill, 2006: cap 40

42. Briggs M. Surgical wound pain: a trial of two treatments. J Wound Care 1996; 5: 456-60.

43. Huxtable K. Ritual cleansing. Nursing New Zealand 1993; 1: 14-6.
44. Lawrence JC. Wound irrigation. Journal of Wound Care 1997; 6: 23-6.

45. Philips D, Davey C. Clinical Corner. Wound cleansing versus wound disinfection: a challenging dilemma. Perspectives 1997; 21: 15-6.

46. Joanna Briggs Institute. Wound Dressing. Adelaide: Joanna Briggs Institute Margaret Graham Building, Royal Adelaide Hospital, North Terrace, 1998.

47. Fernandez R, Griffiths R. Agua para la limpieza de heridas Cochrane Database Syst Rev. 2008; 23 . CD003861.

48. Fowler E. Wound cleansing. Journal of Gerontical Nursing 1985; 11: 42-3.

49. Angeras MH, Brandberg A, Falk A, Seeman T. Comparison between sterile saline and tap water for the cleaning of acute traumatic soft tissue wounds. European Journal of Surgery 1992; 158: 347-50.

50. Murphy A. Wound Care. Cleansing Solutions Nursing Times 1995; 91: 78-80.

51. Thompson S. Towards evidence based emergency medicine: best BETS from the Manchester Royal Infirmary. Wound cleaning methods. Journal of Accident and Emergency Medicine 1999; 16: $63-$

52. Dearden $\mathrm{CH}$. Traumatic wounds: cleaning and dressing. Nursing Times 2001; 97: 50-8.

53. McDonnell G, Russell AD. Antiseptics and Disinfectants Activity, Action, and Resistance. Clinical Microbiol Rev 1999; 12: 147-79.

54. De Vries JH, van Dorp WT, van Barneveld PWC. A randomized trial of alcohol $70 \%$ versus alcoholic iodine $2 \%$ in skin disinfection before insertion of peripheral infusion catheters. J Hosp Infect 1997; 36: 317-20.

55. Graham M. Frequency and duration of handwashing in an intensive care unit. Am J Infect Control 1990; 18: 77-81.

56. Fendler EJ, Dolan MJ, Hammond BS, Williams RA. Effects of topical alcohol gel use on human skin. Infect Control Hosp Epidemiol 2000; 21: 103

57. Crabtree TD, Pelletier SJ, Pruett TL. Surgical antisepsis. In: Block SS, ed. Disinfection, sterilization, and preservation. 5dh ed. Philadelphia,'PA: Lippincott. Williams \& Wilkins; 2001. pp. 91934.

58. Ministerio de Sanidad y Consumo. Vacunación en adultos. Recomendaciones. Vacuna de difteria y tétanos. Madrid: Ministerio de Sanidad y Consumo; 2009. [En línea] [fecha de acceso: 09 de Enero de 2013]. URL disponible en: http://www.msc.es/ciudadanos/proteccionSalud/vacunaciones/docs/TetanosDifteria_2009.pdf. 